\begin{tabular}{|c|l|}
\hline Title & A coustic-phonon cavity modes in one dimensional multilayered el astic structures \\
\hline Author(s) & Tamura, Shin-ichiro; Watanabe, Haruka; Kawasaki, Takashi \\
\hline Citation & $\begin{array}{l}\text { PHY SICAL REVIEW B, 72, 165306 } \\
\text { https://doi.org/L0.1103/PhysRevB.72.165306 }\end{array}$ \\
\hline Issue Date & 2005 \\
\hline Doc URL & http://hdl.handle.net/2115/5915 \\
\hline Rights & Copyright $\odot 2005$ A merican Physical Society \\
\hline Type & article \\
\hline File Information & PRB72-16.pdf \\
\hline
\end{tabular}

Instructions for use 


\title{
Acoustic-phonon cavity modes in one-dimensional multilayered elastic structures
}

\author{
Shin-ichiro Tamura, Haruka Watanabe, and Takashi Kawasaki \\ Department of Applied Physics, Hokkaido University, Sapporo 060-8628, Japan \\ (Received 28 April 2005; revised manuscript received 25 July 2005; published 3 October 2005)
}

\begin{abstract}
We theoretically study "cavity" phonons, i.e., acoustic phonons localized in a foreign layer (a cavity layer) embedded in a periodic one-dimensional superlattice (SL) in the isotropic, continuum approximation. To find the eigenfrequencies of the cavity modes we develop a formulation based on the transfer matrix and Green's function methods and apply it to the case where the confined phonons propagate along the layer interfaces. These cavity phonons are predicted to exist for both the coupled longitudinal and transverse acoustic mode (the sagittal mode) and the single mode with pure transverse polarization (the shear-horizontal mode). Numerical examples are presented for periodic Al/W multilayered structures with a Ag cavity layer and GaAs/AlAs SLs with an $\mathrm{Al}_{0.8} \mathrm{Ga}_{0.2} \mathrm{As}$ layer. Finite-difference time-domain calculations for phonon packet propagation are also conducted to directly illustrate the existence of the confined cavity modes and also to confirm the validity of our formulation.
\end{abstract}

DOI: $10.1103 /$ PhysRevB.72.165306

PACS number(s): 62.25. $+\mathrm{g}, 62.30 .+\mathrm{d}$

\section{INTRODUCTION}

The effects of inhomogeneities on the propagation of phonons and acoustic waves in periodic, elastic multilayered structures or superlattices (SLs) have recently been studied extensively. In particular, the presence and characteristics of localized phonons at the surface of semi-infinite SLs have been studied theoretically with the isotropic approximation ${ }^{1,2}$ and also by including elastic anisotropy. ${ }^{3}$ Experimental studies with the picosecond ultrasonic technique ${ }^{4}$ and Raman scattering have revealed the existence of localized acoustic surface modes in both semiconducting and metallic SLs. ${ }^{5-8}$ Also the localized vibrations at the interface between a superlattice and a substrate have been investigated by DjafariRouhani and co-workers. ${ }^{9,10}$ All these localized modes associated with the inhomogeneities that destroy the perfect periodicity of a system appear inside the band gaps of the bulk modes. Possible applications of these surface and interface "guided modes" to signal-processing devices have been discussed by Murdoch. ${ }^{11}$

Another interesting inhomogeneity is induced by introducing a single or a few layers different from the constituent layers of the host SL. It has been well established that even a small amount of defects in a crystal lattice can radically alter the frequencies of the normal modes of vibration as well as the associated pattern of atomic displacement. ${ }^{12,13}$ Similar effects are also expected to occur when a layer with different thickness or of different material, i.e., a foreign layer (a cavity layer), is introduced in an otherwise perfect SL. More specifically, vibrations localized at this embedded cell appear, in general, with eigenfrequencies inside the frequency gaps of bulk phonons existing at the center, boundaries, and even inside the folded Brillouin zone of the host superlattice. ${ }^{14}$ We call such localized vibrations "cavity" modes of phonons.

Recently, with Raman scattering experiments, Trigo et al. demonstrated the existence of a confined acoustical vibration in a GaAs/AlAs SL with an AlGaAs cavity layer. ${ }^{15,16}$ This vibration was found in a zone-center frequency gap with a complex SL wave number $q_{z}$ (we take the $z$ axis along the growth direction of the SL) and for the vanishing wave vector parallel to the layer interfaces $\mathbf{k}_{\|}=\left(k_{x}, k_{y}\right)=\mathbf{0}$. The associated lattice displacement is, however, expected to be considerably extended on either side of the cavity layer due to small acoustic mismatch between the cavity and host layers. The theoretical studies on the cavity phonons in SLs developed so far are mostly restricted to this case with $\mathbf{k}_{\|}=\mathbf{0}$. $^{22,23}$ For these nonpropagating local modes, three kinds of acoustic vibrations are treated to be decoupled from each other (valid in the isotropic approximation, for example) and accordingly the mathematical analysis is rather straightforward.

A more general and physically appealing case is that the confined modes propagate along the layer interfaces, i.e., $\mathbf{k}_{\|} \neq \mathbf{0}$, with their vibrational amplitudes localized in the cavity cell and its neighborhood. We still call these propagating confined modes the cavity phonons. These phonons are analogous to the waveguide modes in two-dimensional phononic crystals with a line defect. ${ }^{17,18}$ Hence, the existence of propagating cavity phonons should prove that SLs with a foreign layer can work as efficient planar waveguides for phonons and ultrasound, and may have potential applications to acoustic devices such as frequency and wave-vector selective filters, and the acoustic analog of channel drop filters. ${ }^{19,20}$ Also the phonon-phonon and electron-phonon interactions in the cavity layer should be highly enhanced due to the strong confinement effects and the associated large displacement amplitudes. Thus, the cavity phonons with $\mathbf{k}_{\|}$ $\neq \mathbf{0}$ are expected to play important roles in the transport of thermal energy in SLs and multilayered structures. ${ }^{21}$

The purpose of the present paper is to formulate the propagating cavity phonons in SLs. In the isotropic approximation we assume, we can set $\mathbf{k}_{\|}=\left(k_{\|}, 0\right)$. In this case two vibrational modes (longitudinal and transverse modes) polarized inside the $x-z$ plane (the sagittal plane) are coupled to each other and possibly induce the cavity-phonon modes which we call sagittal (SG) cavity modes, while the transverse vibrations polarized in the $y$ direction parallel to the layer interfaces are decoupled from the former mode and induce the cavity modes referred to as the shear-horizontal 
(SH) cavity modes. For the SG cavity mode in SLs we will give explicit formulas which determine their eigenfrequencies, or cavity branches, and the spatial profiles of the associated lattice displacements. For the simpler SH cavity mode the corresponding formulas are given by slightly modifying the results of Ref. 3 for the nonpropagating $\left(\mathbf{k}_{\|}=\mathbf{0}\right)$ mode. So, only relevant notes for $\mathbf{k}_{\|} \neq \mathbf{0}$ are presented in the Appendix.

In the next section, we develop a mathematical formulation for coupled longitudinal (L) and transverse (T) phonons in periodic multilayered structures based on the transfermatrix and Green's function methods. In Sec. III we apply these methods to finding cavity phonon modes in SLs when a foreign layer is introduced. Numerical examples are presented in Sec. IV for an $\mathrm{Al} / \mathrm{W}$ multilayered structure with a $\mathrm{Ag}$ cavity layer and for an AlAs/GaAs SL with an AlGaAs cavity layer. Conclusions are given in Sec. V. In the Appendix we give some notes for the SH cavity-phonon mode.

\section{FORMULATION}

\section{A. Periodic multilayered structures and transfer matrix}

We consider a periodic SL consisting of alternating $A$ and $B$ layers that are stacked in the $z$ direction with a flat interfaces parallel to the $x-y$ plane. The thicknesses of the constituent layers are $d_{A}$ and $d_{B}$, respectively, and the unit period is $D=d_{A}+d_{B}$. For the general case where $A$ and $B$ are anisotropic materials all three modes of vibrations (one quasilongitudinal and two quasitransverse modes) are coupled to each other and the displacement vector $\mathbf{U}_{I, n}$ and the stress vector $\mathbf{S}_{I, n}$ (the component of the stress tensor normal to the interfaces) in the $I$-type layer $(I=A, B)$ of the $n$th period are written as ${ }^{24}$

$$
\begin{aligned}
\left(\begin{array}{c}
\mathbf{U}_{I, n}(\mathbf{x}, t) \\
\mathbf{S}_{I, n}(\mathbf{x}, t)
\end{array}\right) & =\sum_{j=1}^{6} a_{I, n}^{(j)}\left(\begin{array}{c}
\mathbf{e}_{I}^{(j)} \\
\boldsymbol{\sigma}_{I}^{(j)}
\end{array}\right) \exp \left(i k_{I, z}^{(j)} z\right) e^{i\left(\mathbf{k}_{\|} \cdot \mathbf{x}_{\|}-\omega t\right)} \\
& \equiv \mathbf{W}_{I, n}(z) e^{i\left(\mathbf{k}_{\|} \mathbf{x}_{\|}-\omega t\right)} \quad(I=A, B),
\end{aligned}
$$

where $\mathbf{x}=\left(\mathbf{x}_{\|}, z\right)=(x, y, z), j$ is the index which discriminates six waves (three pairs of counterpropagating waves) in a layer, $a$ is the amplitude, $\mathbf{e}$ is the unit polarization vector, $\boldsymbol{\sigma}$ is the stress vector whose components are defined by $\left[\sigma_{I}^{(j)}\right]_{i}$ $=c_{I, i 3 m n} k_{n} e_{m} / k_{\|}$with $c_{I, i l m n}$ the stiffness tensor (the summation convention over repeated indices is assumed), $\mathbf{k}$ $=\left(\mathbf{k}_{\|}, k_{z}\right)=\left(k_{x}, k_{y}, k_{z}\right)=\left(k_{1}, k_{2}, k_{3}\right)$ with $k_{z}=k_{I, z}^{(j)}$ the wave vector, and $\omega$ is the angular frequency. The wave numbers $k_{I z}^{(j)}$ $(j=1-6)$, which are complex in general, are determined by solving the Christoffel equations

$$
\left(\rho_{I} \omega^{2} \delta_{i m}-c_{I, i l m n} k_{l} k_{n}\right) e_{m}=0 \quad(i=1,2,3),
$$

for given $\mathbf{k}_{\|}$(a real vector) and $\omega$, where $\rho$ is the mass density. However, if $A$ and $B$ are isotropic materials the Christoffel equations Eq. (2) are factorized into two sets; one for the pure $\mathrm{T}$ mode polarized in the $y$ direction (the SH mode), and the other for the coupled $\mathrm{L}$ and $\mathrm{T}$ modes polarized inside the $x-z$ plane with $x$ direction parallel to the wave vector $\mathbf{k}_{\|}$ (the SG mode). In the present study we assume this case and the formulation is developed predominantly for the latter SG mode. The results for the former SH mode are briefly noted in the Appendix.

For the SG mode consisting of coupled $\mathrm{L}$ and $\mathrm{T}$ vibrations the sum over $j$ in Eq. (1) extends up to four (two pairs of counterpropagating waves in a layer) instead of six and we can explicitly write the four-component vector $\mathbf{W}_{A, n}(z)$ in the $A$ layer as ${ }^{25,26}$

$$
\mathbf{W}_{A, n}(z)=\hat{\Gamma}_{A} \hat{\Phi}_{A}(z) \mathbf{A}_{n},
$$

where

$$
\begin{aligned}
& \hat{\Gamma}_{A}=\left(\begin{array}{cccc}
\sin \theta_{A}^{(L)} & \cos \theta_{A}^{(T)} & \sin \theta_{A}^{(L)} & -\cos \theta_{A}^{(T)} \\
\cos \theta_{A}^{(L)} & -\sin \theta_{A}^{(T)} & -\cos \theta_{A}^{(L)} & -\sin \theta_{A}^{(T)} \\
2 C_{44}^{A} \cos \theta_{A}^{(L)} & C_{44}^{A} \frac{\cos \left(2 \theta_{A}^{(T)}\right)}{\sin \theta_{A}^{(T)}} & -2 C_{44}^{A} \cos \theta_{A}^{(L)} & C_{44}^{A} \frac{\cos \left(2 \theta_{A}^{(T)}\right)}{\sin \theta_{A}^{(T)}} \\
C_{11}^{A} \frac{\cos \left(2 \theta_{A}^{(T)}\right)}{\sin \theta_{A}^{(L)}} & -2 C_{44}^{A} \cos \theta_{A}^{(T)} & C_{11}^{A} \frac{\cos \left(2 \theta_{A}^{(T)}\right)}{\sin \theta_{A}^{(L)}} & 2 C_{44}^{A} \cos \theta_{A}^{(T)}
\end{array}\right) \\
& \hat{\Phi}_{A}(z)=\left(\begin{array}{cccc}
\exp \left[i k_{A, z}^{(L)} z\right] & 0 & 0 & 0 \\
0 & \exp \left[i k_{A, z}^{(T)} z\right] & 0 & 0 \\
0 & 0 & \exp \left[-i k_{A, z}^{(L)} z\right] & 0 \\
0 & 0 & 0 & \exp \left[-i k_{A, z}^{(T)} z\right]
\end{array}\right) \text {, }
\end{aligned}
$$


and

$$
\mathbf{A}_{n}=\left(a_{A, n}^{(1)}, a_{A, n}^{(2)}, a_{A, n}^{(3)}, a_{A, n}^{(4)}\right)^{t} \equiv\left(a_{A, n}^{(L)}, a_{A, n}^{(T)}, \widetilde{a}_{A, n}^{(L)}, \widetilde{a}_{A, n}^{(T)}\right)^{t} .
$$

In these equations $\theta_{A}^{(L)}$ and $\theta_{A}^{(T)}$ are the propagation angles in the $A$ layer measured from the normal of the interfaces. We have also written $\left(k_{A, z}^{(1)}, k_{A, z}^{(2)}, k_{A, z}^{(3)}, k_{A, z}^{(4)}\right)^{t} \equiv\left(k_{A, z}^{(L)}, k_{A, z}^{(T)},-k_{A, z}^{(L)}\right.$, $\left.-k_{A, z}^{(T)}\right)^{t}$ and the superscripts $L$ and $T$ represent the longitudinal and transverse vibrations. The abbreviated subscript notations, e.g., $C_{44}^{I}=c_{I, 1212}$, etc., have been used for the elastic constants. The corresponding vector $\mathbf{W}_{B, n}(z)$ in the $B$ layer is defined similarly.

Next we define the transfer matrix $\hat{T}_{n}$ which relates $\mathbf{W}_{I, n}$ and $\mathbf{W}_{I, n+1}$ as

$$
\mathbf{W}_{A, n+1}(n D)=\hat{T}_{n} \mathbf{W}_{A, n}[(n-1) D] .
$$

For a perfect periodic system $\hat{T}_{n}$ is independent of $n$, or $\hat{T}_{n}$ $=\hat{T}$, and we find

$$
\mathbf{W}_{A, n+1}(n D)=(\hat{T})^{n} \mathbf{W}_{A, 1}(0) .
$$

There exist similar equations with $A$ replaced by $B$ and the explicit expression for $\hat{T}$ is given by $\hat{T}=\hat{T}_{B} \hat{T}_{A}$ with

$$
\hat{T}_{I}=\hat{\Gamma}_{I} \hat{\Phi}_{I}\left(d_{I}\right) \hat{\Gamma}_{I}^{-1} \quad(I=A, B) .
$$

\section{B. Green's tensor in periodic superlattices}

We try to find the lattice displacement $\mathbf{U}_{I, n}$ at the layer interfaces by introducing the Green's tensor in periodic superlattices. To save the indices we define $\mathbf{W}_{A, n+1}(n D) \equiv \mathbf{w}_{n}$ and hence

$$
\mathbf{w}_{n+1}=\hat{T}_{n} \mathbf{w}_{n},
$$

where

$$
\mathbf{w}_{n}=\left(\begin{array}{l}
\mathbf{U}_{n} \\
\mathbf{S}_{n}
\end{array}\right)
$$

with $\mathbf{U}_{n} \equiv \mathbf{U}_{A, n+1}(n D)$, etc., and

$$
\hat{T}_{n}=\left(\begin{array}{ll}
\hat{T}_{n}^{(1)} & \hat{T}_{n}^{(2)} \\
\hat{T}_{n}^{(3)} & \hat{T}_{n}^{(4)}
\end{array}\right) .
$$

In Eq. (12) $\hat{T}_{n}^{(i)}(i=1-4)$ are $2 \times 2$ matrices.

Eliminating $\mathbf{S}_{n}$ from Eq. (10), we obtain an equation that relates the lattice displacements at the interfaces of adjacent unit cells,

$$
\hat{K}_{n} \mathbf{U}_{n+1}+\hat{J}_{n-1} \mathbf{U}_{n-1}=\left(\hat{M}_{n}+\hat{N}_{n-1}\right) \mathbf{U}_{n}
$$

where

$$
\begin{gathered}
\hat{K}_{n}=\left(\hat{T}_{n}^{(2)}\right)^{-1}, \\
\hat{J}_{n}=\hat{T}_{n}^{(4)}\left(\hat{T}_{n}^{(2)}\right)^{-1} \hat{T}_{n}^{(1)}-\hat{T}_{n}^{(3)}, \\
\hat{M}_{n}=\left(\hat{T}_{n}^{(2)}\right)^{-1} \hat{T}_{n}^{(1)},
\end{gathered}
$$

$$
\hat{N}_{n}=\hat{T}_{n}^{(4)}\left(\hat{T}_{n}^{(2)}\right)^{-1}
$$

In the perfect, periodic SL consisting of alternating $A$ and $B$ layers, matrices $\hat{K}, \hat{J}, \hat{M}$, and $\hat{N}$ are independent of $n$ and hence on deleting this index Eq. (13) is formally written as

$$
\hat{L}_{n m} \mathbf{U}_{m}=\mathbf{0} \text {, }
$$

with

$$
\hat{L}_{n m}=\hat{K} \delta_{n+1, m}+\hat{J} \delta_{n-1, m}-\hat{R} \delta_{n, m}
$$

and

$$
\hat{R}=\hat{M}+\hat{N}
$$

Now we introduce the Green's function $\hat{G}_{n m}$ defined by

$$
\hat{L}_{n l} \hat{G}_{l m}=\delta_{n, m} \hat{I},
$$

where $\hat{\mathrm{I}}$ is a unit matrix. In order to solve this equation, we expand $\hat{G}_{n m}$ in a Fourier series,

$$
\hat{G}_{n m}=\sum_{q} e^{i q x_{n}} \widetilde{g}_{m}(q)
$$

where $x_{n}=n D$ and the wave number $q$ is restricted to $-\pi / D \leqslant q \leqslant \pi / D$, i.e., the mini-Brillouin zone. Noting that

$$
\delta_{n, m}=\frac{1}{N_{0}} \sum_{q} e^{i q\left(x_{n}-x_{m}\right)},
$$

with $N_{0}$ the number of periods, we obtain

$$
\tilde{g}_{m}(q)=\frac{1}{N_{0}}\left(\hat{K} e^{i q D}+\hat{J} e^{-i q D}-\hat{R}\right)^{-1} e^{-i q x_{m}} .
$$

Thus, by converting the sum over $q$ into the integral over the mini-Brillouin zone according to

$$
\frac{1}{N_{0}} \sum_{q} \rightarrow \frac{D}{2 \pi} \int_{-\pi / D}^{\pi / D} d q
$$

the Green's function [Eq. (22)] is expressed as

$$
\hat{G}_{n m}=\frac{D}{2 \pi} \int_{-\pi / D}^{\pi / D}\left(\hat{K} e^{i q D}+\hat{J} e^{-i q D}-\hat{R}\right)^{-1} e^{i q(n-m) D} d q .
$$

From this equation we see that $\hat{G}_{n m}=\hat{G}_{n-m}$. The integral over $q$ can be done analytically. To see this we first write Eq. (26) as

$$
\begin{aligned}
\hat{G}_{n}= & \frac{1}{2 \pi} \int_{-\pi}^{\pi} d \varphi\left(\hat{K} e^{i \varphi}+\hat{J} e^{-i \varphi}-\hat{R}\right)^{-1} e^{i n \varphi} \\
& =\frac{1}{2 \pi} \int_{-\pi}^{\pi} d \varphi \frac{1}{\Delta\left(e^{i \varphi}\right)} \hat{\Xi}\left(e^{i \varphi}\right) e^{i n \varphi},
\end{aligned}
$$

where

$$
\Delta\left(e^{i \varphi}\right) \equiv \operatorname{det}\left|\hat{K} e^{i \varphi}+\hat{J} e^{-i \varphi}-\hat{R}\right|
$$




$$
=\operatorname{det}\left(\begin{array}{cc}
2 K_{11} \cos \varphi-R_{11} & \beta \cos \varphi-R_{12}+i \gamma \sin \varphi \\
-\beta \cos \varphi+R_{12}+i \gamma \sin \varphi & 2 K_{22} \cos \varphi-R_{22}
\end{array}\right)
$$

and

$$
\begin{aligned}
\hat{\Xi}\left(e^{i \varphi}\right)= & \left(\begin{array}{cc}
2 K_{22} \cos \varphi-R_{22} & -\beta \cos \varphi+R_{12} \\
\beta \cos \varphi-R_{12} & 2 K_{11} \cos \varphi-R_{11}
\end{array}\right) \\
& -i \gamma \sin \varphi\left(\begin{array}{ll}
0 & 1 \\
1 & 0
\end{array}\right),
\end{aligned}
$$

with $\beta=K_{12}-K_{21}$ and $\gamma=K_{12}+K_{21}$. In deriving the expressions of Eqs. (30) and (31), we have used the following equations deduced with the symmetry properties of the transfer matrix $\hat{T}$ :

$$
\begin{gathered}
\hat{K}=\left(\begin{array}{ll}
K_{11} & K_{12} \\
K_{21} & K_{22}
\end{array}\right), \\
\hat{J}=\left(\begin{array}{cc}
K_{11} & -K_{21} \\
-K_{12} & K_{22}
\end{array}\right), \\
\hat{R}=\left(\begin{array}{cc}
R_{11} & R_{12} \\
-R_{12} & R_{22}
\end{array}\right) .
\end{gathered}
$$

Here, it should be noted that $\Delta\left(e^{i \varphi}\right)=0$ gives the dispersion relations of the coupled $\mathrm{L}$ and $\mathrm{T}$ phonons in the perfect, periodic superlattice. Hence, $\Delta\left(e^{i \varphi}\right)$ is factorized as

$$
\Delta\left(e^{i \varphi}\right)=\alpha e^{-2 i \varphi}\left(e^{i \varphi}-z_{1}\right)\left(e^{i \varphi}-z_{2}\right)\left(e^{i \varphi}-\frac{1}{z_{1}}\right)\left(e^{i \varphi}-\frac{1}{z_{2}}\right)
$$

where $\alpha \equiv \operatorname{det} \hat{K}$ and $z_{1}$ and $z_{2}$ (also $1 / z_{1}$ and $1 / z_{2}$ ) are eigenvalues of the transfer matrix $\hat{T}$. In a frequency band of the coupled L and T phonons, at least either $\left|z_{1}\right|=1$ or $\left|z_{2}\right|=1$ is satisfied, but inside the mutual frequency gaps of these phonons two of the eigenvalues (we choose $z_{1}$ and $z_{2}$ ) have modulus smaller than unity, or $\left|z_{1}\right|<1$ and $\left|z_{2}\right|<1$. We are interested in the latter case because the eigenfrequencies of the cavity modes are found inside those mutual frequency gaps of the host superlattice.

Thus, the Green's function is calculated to be

$$
\begin{aligned}
\hat{G}_{n}= & \frac{1}{2 \pi}\left[\left(\begin{array}{cc}
2 K_{22} & -\beta \\
\beta & 2 K_{11}
\end{array}\right) I_{n}^{(1)}-\left(\begin{array}{cc}
R_{22} & -R_{12} \\
R_{12} & R_{11}
\end{array}\right) I_{n}^{(2)}\right. \\
& \left.+\left(\begin{array}{ll}
0 & \gamma \\
\gamma & 0
\end{array}\right) I_{n}^{(3)}\right],
\end{aligned}
$$

where $I_{n}^{(1)}, I_{n}^{(2)}$, and $I_{n}^{(3)}$ are given by

$$
\begin{gathered}
I_{n}^{(1)} \equiv \int_{-\pi}^{\pi} d \varphi \frac{1}{\Delta\left(e^{i \varphi}\right)} \cos \varphi \cos |n| \varphi \\
=\frac{\pi}{\alpha} \frac{z_{1} z_{2}}{\left(z_{1}-z_{2}\right)\left(z_{1} z_{2}-1\right)}\left(\frac{z_{1}^{2}+1}{z_{1}^{2}-1} z_{1}^{|n|}-\frac{z_{2}^{2}+1}{z_{2}^{2}-1} z_{2}^{|n|}\right),
\end{gathered}
$$

$$
I_{n}^{(2)} \equiv \int_{-\pi}^{\pi} d \varphi \frac{1}{\Delta\left(e^{i \varphi}\right)} \cos |n| \varphi
$$

$$
\begin{gathered}
=\frac{2 \pi}{\alpha} \frac{z_{1} z_{2}}{\left(z_{1}-z_{2}\right)\left(z_{1} z_{2}-1\right)}\left(\frac{z_{1}^{|n|+1}}{z_{1}^{2}-1}-\frac{z_{2}^{|n|+1}}{z_{2}^{2}-1}\right), \\
I_{n}^{(3)} \equiv \int_{-\pi}^{\pi} d \varphi \frac{1}{\Delta\left(e^{i \varphi}\right)} \sin \varphi \sin n \varphi \\
=-\frac{\pi}{\alpha} \frac{z_{1} z_{2}}{\left(z_{1}-z_{2}\right)\left(z_{1} z_{2}-1\right)}\left(z_{1}^{|n|}-z_{2}^{|n|}\right) .
\end{gathered}
$$

Accordingly, the Green's function $\hat{G}_{n}$ decreases exponentially as $|n|$ increases.

\section{CAVITY PHONONS}

We consider the structure with a cavity layer $(C)$ of thickness $d_{C}$ situated at the center $(n=0)$ of the periodic multilayers. In this case the relevant transfer matrices are

$$
\hat{T}_{n}=\left\{\begin{array}{cc}
\hat{T}_{C} & (n=0), \\
\hat{T} & (n \neq 0),
\end{array}\right.
$$

and the equations governing the lattice displacements are

$$
\begin{gathered}
\left(\hat{K}+\delta \hat{K} \delta_{n, 0}\right) \mathbf{U}_{n+1}+\left(\hat{J}+\delta \hat{J} \delta_{n, 1}\right) \mathbf{U}_{n-1} \\
=\left[\hat{R}+\left(\delta \hat{M} \delta_{n, 0}+\delta \hat{N} \delta_{n, 1}\right)\right] \mathbf{U}_{n},
\end{gathered}
$$

where $\hat{T}_{C}$ is the transfer matrix associated with the cavity layer [Eq. (9) with $I=C$ ] and

$$
\begin{gathered}
\delta \hat{K}=\left(\hat{T}_{C}^{(2)}\right)^{-1}-\hat{K}, \\
\delta \hat{J}=\left[\hat{T}_{C}^{(4)}\left(\hat{T}_{C}^{(2)}\right)^{-1} \hat{T}_{C}^{(1)}-\hat{T}_{C}^{(3)}\right]-\hat{J}, \\
\delta \hat{M}=\left(\hat{T}_{C}^{(2)}\right)^{-1} \hat{T}_{C}^{(1)}-\hat{M}, \\
\delta \hat{N}=\hat{T}_{C}^{(4)}\left(\hat{T}_{C}^{(2)}\right)^{-1}-\hat{N} .
\end{gathered}
$$

Next we introduce the matrix $\delta \hat{L}_{n m}$ defined by

$$
\delta \hat{L}_{n m}=\delta \hat{K} \delta_{n, 0} \delta_{n, m-1}+\delta \hat{J} \delta_{n, 1} \delta_{n, m+1}-\left(\delta \hat{M} \delta_{n, 0}+\delta \hat{N} \delta_{n, 1}\right) \delta_{n, m} .
$$

With this matrix, the equations of motion become

$$
\left(\hat{L}_{n m}+\delta \hat{L}_{n m}\right) \mathbf{U}_{m}=\left(\delta_{n, m}+\hat{G}_{n l} \delta \hat{L}_{l m}\right) \mathbf{U}_{m}=\mathbf{0},
$$

where

$$
\delta \hat{L} \equiv\left(\delta \hat{L}_{l m}\right)=\left(\begin{array}{ccc}
\hat{0} & \hat{0} & \hat{0} \\
\hat{0} & \delta \hat{l} & \hat{0} \\
\hat{0} & \hat{0} & \hat{0}
\end{array}\right)
$$




$$
\hat{G} \equiv\left(\hat{G}_{n m}\right)=\left(\begin{array}{ccc}
\widetilde{G}_{11} & \hat{G}_{-} & \widetilde{G}_{13} \\
\hat{G}_{+}^{\prime} & \hat{G}_{C} & \hat{G}_{-}^{\prime} \\
\widetilde{G}_{31} & \hat{G}_{+} & \widetilde{G}_{33}
\end{array}\right),
$$

and the nonvanishing component $\delta \hat{l}$ of $\delta \hat{L}_{n m}$ consists of a $4 \times 4$ matrix given by

$$
\delta \hat{l}=\left(\begin{array}{cc}
-\delta \hat{M} & \delta \hat{K} \\
\delta \hat{J} & -\delta \hat{N}
\end{array}\right) .
$$

Also the elements of the Green's tensors are

$$
\begin{gathered}
\hat{G}_{-}=\left(\begin{array}{cc}
\hat{G}_{1-N} & \hat{G}_{-N} \\
\hat{G}_{2-N} & \hat{G}_{1-N} \\
\vdots & \vdots \\
\hat{G}_{-2} & \hat{G}_{-3} \\
\hat{G}_{-1} & \hat{G}_{-2}
\end{array}\right), \\
\hat{G}_{+}=\left(\begin{array}{cc}
\hat{G}_{2} & \hat{G}_{1} \\
\hat{G}_{3} & \hat{G}_{2} \\
\vdots & \vdots \\
\hat{G}_{N-1} & \hat{G}_{N-2} \\
\hat{G}_{N} & \hat{G}_{N-1}
\end{array}\right),
\end{gathered}
$$

and

$$
\hat{G}_{C}=\left(\begin{array}{cc}
\hat{G}_{0} & \hat{G}_{-1} \\
\hat{G}_{1} & \hat{G}_{0}
\end{array}\right) .
$$

The other entries in Eq. (52) are irrelevant to the further calculations and hence their expressions are not given here. Finally, introducing the displacement vectors defined by

$$
\begin{gathered}
\mathbf{v} \equiv\left(\begin{array}{c}
\mathbf{U}_{-N+1} \\
\vdots \\
\mathbf{U}_{-1}
\end{array}\right), \\
\mathbf{u} \equiv\left(\begin{array}{c}
\mathbf{U}_{0} \\
\mathbf{U}_{1}
\end{array}\right), \\
\mathbf{w} \equiv\left(\begin{array}{c}
\mathbf{U}_{2} \\
\vdots \\
\mathbf{U}_{N}
\end{array}\right),
\end{gathered}
$$

the equations determining the SG cavity modes are summarized as

$$
\begin{gathered}
\mathbf{v}+\hat{G}_{-} \delta \hat{l} \mathbf{u}=\mathbf{0}, \\
\left(\hat{I}+\hat{G}_{C} \delta \hat{l}\right) \mathbf{u}=\mathbf{0},
\end{gathered}
$$

TABLE I. The numerical values employed for the mass density $\rho$ (in units of $\mathrm{g} \mathrm{cm}^{-3}$ ), bulk longitudinal and transverse sound velocities $v_{l}$ and $v_{t}$ (in units of $10^{5} \mathrm{~cm} \mathrm{~s}^{-1}$ ), and acoustic impedances $Z_{l}=\rho v_{l}$ and $Z_{t}=\rho v_{t}$ (in units of $10^{5} \mathrm{~g} \mathrm{~cm}^{-2} \mathrm{~s}^{-1}$ ) tabulated for each material.

\begin{tabular}{cccccc}
\hline \hline $\mathrm{I}$ & $\rho$ & $v_{l}$ & $v_{t}$ & $Z_{l}$ & $Z_{t}$ \\
\hline $\mathrm{Al}$ & 2.73 & 6.37 & 3.09 & 17.41 & 8.45 \\
$\mathrm{~W}$ & 19.32 & 5.21 & 2.88 & 100.6 & 55.6 \\
$\mathrm{Ag}$ & 10.64 & 3.74 & 1.78 & 39.8 & 18.9 \\
$\mathrm{GaAs}$ & 5.32 & 5.03 & 3.03 & 26.8 & 16.1 \\
$\mathrm{AlAs}$ & 3.76 & 5.98 & 3.60 & 22.5 & 13.5 \\
$\mathrm{Al}_{0.8} \mathrm{Ga}_{0.2} \mathrm{As}$ & 4.07 & 5.75 & 3.46 & 23.4 & 14.1 \\
\hline \hline \multicolumn{5}{c}{$\mathbf{w}+\hat{G}_{+} \delta \hat{l} \mathbf{u}=\mathbf{0 .}$} \\
\end{tabular}

The eigenfrequencies $\omega_{C}$ of those cavity modes are obtained as the solutions of the secular equation

$$
\operatorname{det}\left|\hat{I}+\hat{G}_{C} \delta \hat{l}\right|=0
$$

As we shall see in the next section, the eigenfrequencies $\omega_{C}$ for a given $\mathbf{k}_{\|}$are found inside the complete gaps of the host superlattice, where $\left|z_{1}\left(\mathbf{k}_{\|}, \omega_{C}\right)\right|<1$ and $\left|z_{2}\left(\mathbf{k}_{\|}, \omega_{C}\right)\right|<1$ are satisfied simultaneously. In addition, from Eq. (60), the associated lattice displacements at $\omega=\omega_{C}$ are seen to decrease exponentially on either side of the cavity layer. These are analogous to the effects of a defect introduced to perfect, periodic lattices. ${ }^{12,13}$

\section{NUMERICAL EXAMPLES}

\section{A. Al/W superlattices with an Ag cavity layer}

The first example we study is a metallic Al/W multilayered structure $(A=\mathrm{Al}, B=\mathrm{W})$ which exhibits large frequency gaps due to large acoustic mismatch between the constituents aluminum and tungsten as tabulated in Table I. This structure with a foreign layer inserted is expected to show strong cavity effects for phonons. As a possible cavity layer we choose silver $(C=\mathrm{Ag})$ with the magnitudes of acoustic impedances $Z_{l}$ and $Z_{t}$ in between those of aluminum and tungsten (see also the Table I).

Figure 1(a) illustrates the band structure of the periodic $\mathrm{Al} / \mathrm{W}$ superlattice with $d_{\mathrm{Al}}=d_{\mathrm{W}}$ (unit period is $D=d_{\mathrm{Al}}+d_{\mathrm{W}}$ ) together with the cavity branches originating from an embedded Ag layer with $d_{C}=d_{\mathrm{Ag}}=D / 4$ (the thickness is a half of the host layers). The cavity branches of the coupled $\mathrm{L}$ and $\mathrm{T}$ modes (the SG modes) and also of the pure T modes (the SH modes) are shown by the bold and thin lines, respectively. As expected, the frequencies of the cavity modes are found inside the band gaps of the corresponding bulk modes. Here we note that in the band regions of the coupled $\mathrm{L}$ and $\mathrm{T}$ phonons (L-T band) in the original superlattice shown in Fig. 1(a) (also for a GaAs/AlAs SL shown below), at least one of two vibrational eigenfrequencies is real valued.

Figure 1(b) displays the profiles normal to the layer interfaces of the displacement components $u_{x}$ and $u_{z}$ of the SG 


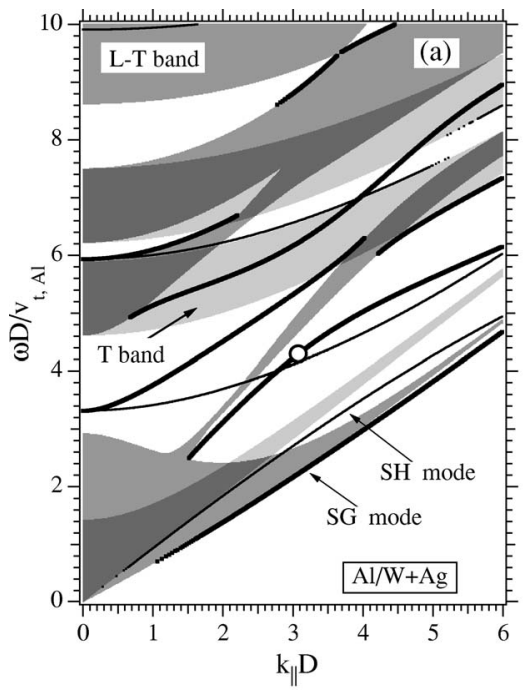

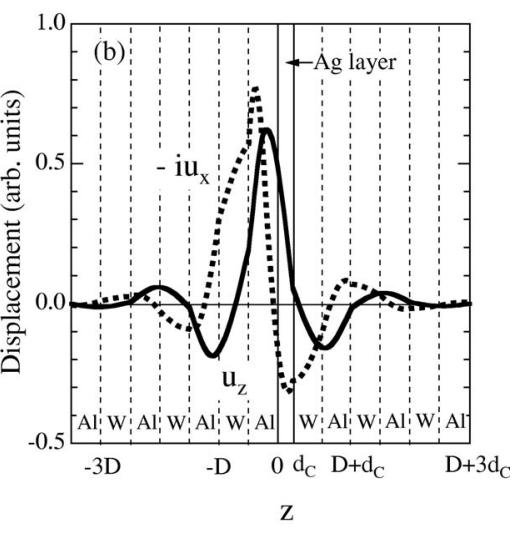

FIG. 1. (a) Band structures and cavity mode branches in the $\mathrm{Al} / \mathrm{W}$ superlattice with $d_{\mathrm{Al}}=d_{\mathrm{W}}$. The cavity layer is silver with thickness $d_{\mathrm{Ag}}$ $=d_{\mathrm{Al}} / 2$ sandwiched between $\mathrm{W}-\mathrm{Al}$ unit cells (the local structure is $\mathrm{W}-\mathrm{Al}-\mathrm{Ag}-\mathrm{W}-\mathrm{Al}$ ). Each band is discriminated according to the darkness, i.e., the lightest band is the pure $\mathrm{T}(\mathrm{SH})$ band, the second lightest band is the coupled L-T (SG) band, and the darkest band is the overlapping of both bands. The SG and SH cavity phonon branches are shown by the bold and thin lines, respectively. (b) Displacement profiles at the point marked by the open circle $\left(k_{\|} D=3\right.$ and $\omega D / v_{t, \mathrm{Al}}=4.22$ ). Bold line is $u_{z}$ (normal to the layer interfaces) and dotted line is $-i u_{x}$ (parallel to the layer interfaces) calculated with analytical formulas. cavity mode at the point marked by the open circle in Fig. 1(a) (at $k_{\|} D=3$ and $\left.\omega D / v_{t, \mathrm{Al}}=4.22\right)$. We recognize that the lattice displacements are well localized near the Ag cell but their profiles are not symmetric because the present multilayered system has an asymmetry under the inversion of $z$. Indeed, for both $u_{x}$ and $u_{z}$ the largest amplitudes are found inside the Al layer adjacent to the Ag layer, which is the lightest element of the structure. Thus, we see as if the pair of the layers composed of silver and its neighboring aluminum act as a cavity cell.

This suggest that more readily understandable results should be obtained when the assumed structure is symmetric with respect to the silver layer. Such a symmetric structure can be realized, for example, if a pair of layers composed of silver and aluminum with the thickness of the latter same as the host layer $d_{\mathrm{Al}}$ are inserted. In this case the Ag layer is sandwiched in between $\mathrm{Al}$ layers and the relevant transfer matrix for the cavity cell is $\hat{T}_{C}=\hat{T}_{\mathrm{Ag}} \hat{T}_{A=\mathrm{Al}}$ with $\hat{T}_{\mathrm{Ag}}$ the transfer matrix for the Ag layer. Figure 2(a) illustrates the cavity branches originated from the Ag layers with $d_{C}=d_{\mathrm{Ag}}$ $=d_{\mathrm{Al}} / 2=D / 4$ (the same thickness for the Ag layer as Fig. 1). Here we still use $d_{C}$ for the thickness of Ag layer. An interesting result is the fact that there exists a cavity branch with vanishing group velocity for a finite $k_{\|}$. Figures 2(b) and 2(c) show how these cavity branches change as the thickness $d_{\mathrm{Ag}}$ of the Ag layer is varied. As $d_{\mathrm{Ag}}$ is increased, the number of the cavity branches is increased. This is because the frequencies associated with the confined vibrational motions in the normal direction become lowered as the cavity layer thickness becomes larger and, at the same time, the intervals of those "quantized" frequencies are decreased. In order to see these results explicitly, we have marked in Figs. 2(a)-2(c)
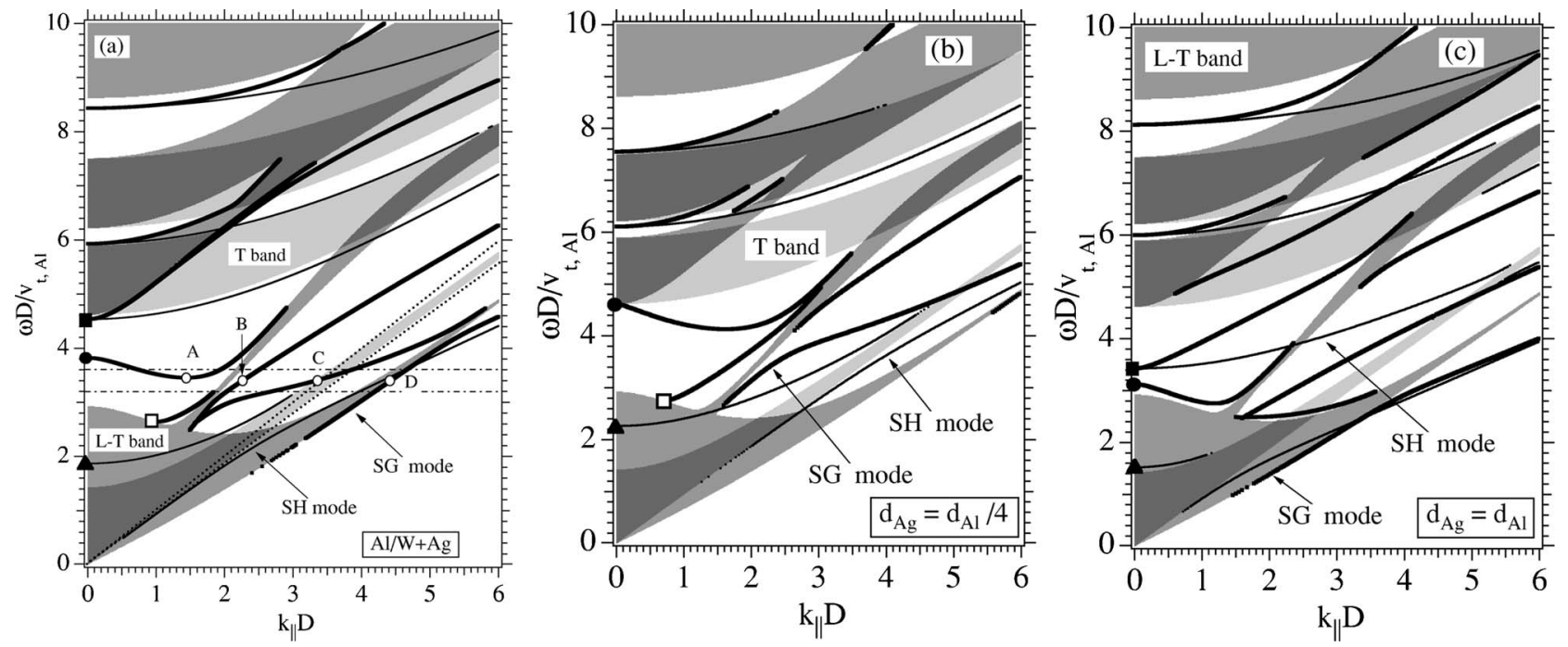

FIG. 2. Band structures in the $\mathrm{Al} / \mathrm{W}$ superlattice with $d_{\mathrm{Al}}=d_{\mathrm{W}}$ the same as in Fig. 1(a) and cavity mode branches. The cavity layer is silver and sandwiched between $\mathrm{W}-\mathrm{Al}$ and $\mathrm{Al}-\mathrm{W}$ unit cells (the local structure is $\mathrm{W}-\mathrm{Al}-\mathrm{Ag}-\mathrm{Al}-\mathrm{W}$ ). The thickness of the the $\mathrm{Ag}$ layer is $d_{\mathrm{Ag}}=(\mathrm{a}) d_{\mathrm{Al}} / 2$, (b) $d_{\mathrm{Al}} / 4$, and (c) $d_{\mathrm{Al}}$. The SG and SH cavity phonon branches are shown by the bold and thin lines, respectively. The edges of the corresponding branches are marked by filled squares, circles, triangles, and open squares. In the FDTD simulations a frequency distribution confined in two dot-dashed lines of (a) is assumed for the Fourier components of the external force $f_{i}$ [Eqs. (62)-(65) and (67)]. In (a) the upper and lower dotted lines show the dispersion relations of the bulk transverse waves in aluminum and tungsten, respectively. 

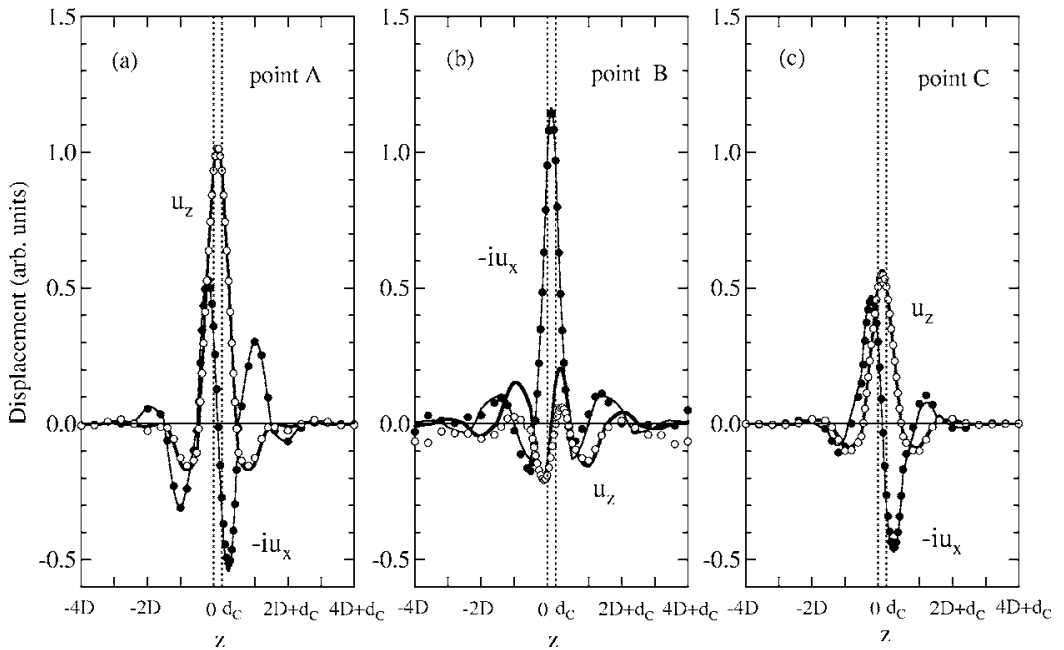

FIG. 3. Displacement profiles in the $\mathrm{Al} / \mathrm{W}$ multilayers with a Ag cavity layer [the same as for Fig. 2(a)]. Bold lines are $u_{z}$ (normal to the layer interfaces) and thin lines are $-i u_{x}$ (parallel to the layer interfaces) calculated with analytical formulas for the points (a) $A\left(k_{\|} D=1.44\right)$, (b) $B\left(k_{\|} D=2.26\right)$, and (c) $C\left(k_{\|} D=3.33\right)$ shown in Fig. 2(a). Open circles and dots are the profiles of $u_{z}$ and $-i u_{x}$ obtained by the FDTD calculations (the profiles along the lines $A^{\prime}, B^{\prime}$, and $C^{\prime}$ in Fig. 5 below). The boundaries of the Ag layer are indicated by vertical dashed lines. the edges of the corresponding cavity branches.

Figure 3 displays the spatial profiles of the displacement components of the SG cavity modes at the points $A$ to $C$ with frequency $\omega D / v_{t, \mathrm{Al}}=3.4\left(\omega D / v_{t, \mathrm{Al}}=3.45\right.$ for the point $\left.A\right)$ indicated in Fig. 2(a). [The relative magnitudes of the displacements in this figure are determined so that $\left|\mathbf{C}_{n=0}\right|=1$ with $\mathbf{C}_{n}=\left(a_{C, n}^{(1)}, a_{C, n}^{(2)}, a_{C, n}^{(3)}, a_{C, n}^{(4)}\right)^{t}$; cf., Eqs. (3) and (6).] The displacement profiles exhibit the expected symmetries with respect to the center of the Ag layer. Also we find that there exists a phase difference of $\pi / 2$ between the displacement components $u_{x}$ and $u_{z}$ parallel to and normal to the interfaces, respectively. (We have assigned the phase so that $u_{z}$ may be real and $u_{x}$ pure imaginary.) Hence, the particle motions associated with the cavity modes are elliptic as the surface localized acoustic waves (Rayleigh waves) in a homogeneous half space. $^{27}$

We see that at the points $A$ and $C, u_{x}$ along the interface is odd with respect to the center of the Ag layer but $u_{z}$ normal to the interfaces is even. Interestingly, these properties for $u_{x}$ and $u_{z}$ are interchanged at the point $B$. Thus, the motions of the $\mathrm{Ag}$ layer at the points $A$ and $C$ are flexural and it is dilatational at the point $B$. The lattice displacements at these points are well localized (within two unit periods or so) on either side of the Ag layer.

The displacement profiles at the point $D$ shown in Fig. 4 exhibit another interesting behavior. Comparing with the lattice displacements at the points $A$ to $C$, they are quite extended due to the fact that this cavity mode frequency is situated close to a bulk band. Here we note that the latter bulk band is located below the $\mathrm{T}$ phonon branches of the constituents materials (aluminum and tungsten) plotted by the dotted lines in Fig. 2(a). Accordingly, this extra frequency band found in the Al/W superlattice originates from the phonons localized at the interfaces, that is, both $k_{A=\mathrm{Al}, z}^{(j)}$ and $k_{B=\mathrm{W}, z}^{(j)}(j=1,4)$, are all complex numbers. Owing to the finite thicknesses of the constituent layers, these localized lattice displacements are extended over neighboring periods and form a frequency band. The existence of this band has already been recognized and it is called the Stoneley band. $^{2,28}$ Note that the phonons or acoustic waves localized on either side of the layer interfaces are reduced to the Stoneley interface waves when the layer thicknesses become infinite. ${ }^{29}$ The spatial amplitude profiles shown in Fig. 4 exhibits the behaviors characteristic of the Stoneley wave at a single interface of aluminum and tungsten as illustrated in the inset. This Stoneley wave or Stoneley band is allowed to exist in restricted regions in the space spanned by the ratios of elastic constants $C_{44}^{A} / C_{44}^{B}$ and mass densities $\rho_{A} / \rho_{B}$ of the constituent materials. ${ }^{30-32}$ No such localized interface mode is found at the interface between bulk GaAs and AlAs.

In order to see the propagation of these cavity modes, we have also made finite-difference time-domain (FDTD) simulations for the time evolution of phonon packets consisting of a narrow range of frequencies. ${ }^{33-35}$ In the FDTD scheme the equations of motion for the lattice displacement

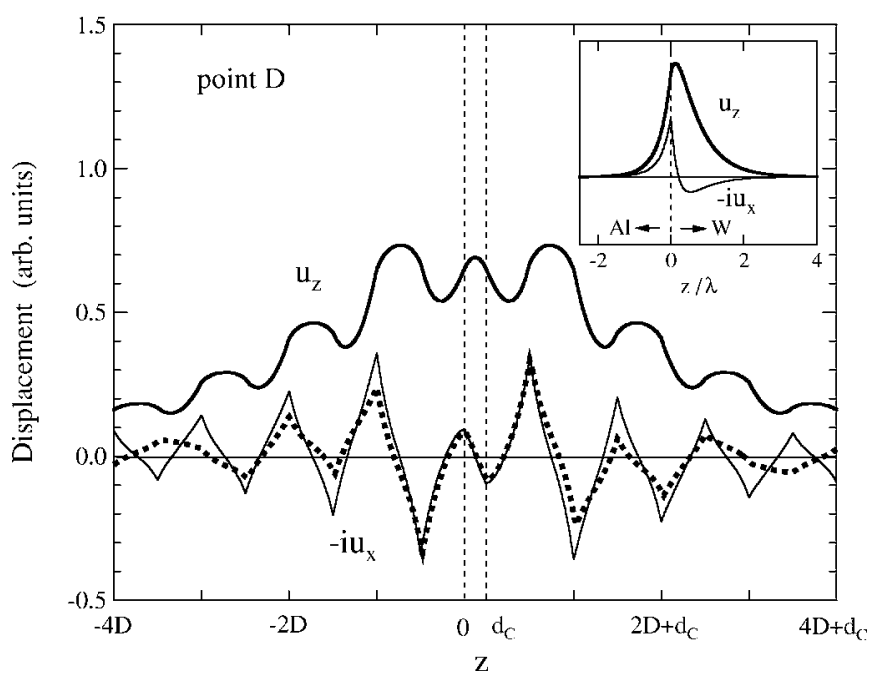

FIG. 4. Displacement profiles at the point $D\left(k_{\|} D=4.43\right)$ in the $\mathrm{Al} / \mathrm{W}$ multilayers with a Ag cavity layer shown in Fig. 2(a). Bold line is $u_{z}$ (normal to the layer interfaces) and thin line is $-i u_{x}$ (parallel to the layer interfaces) calculated with analytical formulas. The dotted line is $-i u_{x}$ obtained by FDTD simulations [the profiles along the dashed line $D^{\prime}$ shown in Fig. 6(c) below]. The boundaries of the Ag layer are indicated by vertical dashed lines. Inset shows the calculated displacement profiles of the Stoneley interface wave at the flat boundary of bulk aluminum and tungsten (distance $z$ is normalized by the wavelength $\lambda$ ). 


$$
\rho(z) \ddot{u}_{i}-c_{i l m n}(z) \partial_{l} \partial_{n} u_{m}=\rho(z) f_{i}(x=0, z, t) \quad(i=1,2,3)
$$

are solved numerically under suitable boundary conditions at the layer interfaces, where $\rho(z)\left[c_{i l m n}(z)\right]$ takes either $\rho_{A}\left(c_{A, i l m n}\right)$ or $\rho_{B}\left(c_{B, i l m n}\right)$ depending on the position $z$. The external force $\mathbf{f}$ applied at $x=0$ has been chosen as

$$
\begin{aligned}
& f_{1}= f_{x}=\left[\Theta\left(z^{\prime}+d_{\mathrm{Ag}} / 3\right)-\Theta\left(z^{\prime}-d_{\mathrm{Ag}} / 3\right)\right] \sin \left[\pi\left(\frac{40}{3 D} z^{\prime}+\frac{1}{4}\right)\right] \\
& \times g(t), \\
& f_{2}=f_{y}=0, \\
& f_{3}=f_{z}=\left[\Theta\left(z^{\prime}+d_{\mathrm{Ag}} / 3\right)-\Theta\left(z^{\prime}-d_{\mathrm{Ag}} / 3\right)\right] \cos \left[\pi\left(\frac{40}{3 D} z^{\prime}+\frac{1}{4}\right)\right] \\
& \times g(t),
\end{aligned}
$$

where $z^{\prime}=z-d_{C} / 2, \Theta(z)$ is a unit step function, i.e., $\Theta(z)$ $=1$ if $z \geqslant 0$ and $\Theta(z)=0$ if $z<0$, and a Gaussian profile in the frequency space with its peak at $\omega=\omega_{0}$ and a full width at half maximum $\Delta \omega$ has been assumed for $g(t)$. Explicitly,

$$
g(t)=g_{0} \exp \left[-(\Delta \omega)^{2}\left(t-t_{0}\right)^{2} / 4\right] \sin \left[\omega_{0}\left(t-t_{0}\right)\right],
$$

with $g_{0}$ a constant.

Figures 5 and 6 exhibit the simulated results for the transverse $\left(u_{z}\right)$ and the longitudinal $\left(u_{x}\right)$ components of phonon packets. In the simulations we have chosen $\omega_{0} D / v_{t, \mathrm{Al}}=3.4$ and $\Delta \omega D / v_{t, \mathrm{Al}}=0.4$ [Fig. 2(a)] and the external force has been given in a line of length $0.6 d_{\mathrm{Ag}}$ normal to the layer interfaces at $x=0$ inside the $\mathrm{Ag}$ layer. The direction of the force exerted is $45^{\circ}$ rotated away from both the $x$ and $z$ axes in the $x-z$ plane. The assumed unit time step for the simulation is $\Delta t=D /\left(200 v_{t, \mathrm{Al}}\right)$ and the maximum of the initial packet is realized at $x=z=0$ at $t=t_{0}=2500 \Delta t=12.5 \mathrm{D} / v_{t, \mathrm{Al}}$ after starting the simulation at $t=0$. Hence, $t=t_{0}$ effectively acts as the origin of the time domain.

Shortly after the excitation the waves in frequency bands propagate in various directions with their characteristic group velocities larger than those of cavity phonons [cf. Fig. 2(a)]. As time elapses we can recognize that the waves confined in the vicinity of the Ag layer propagate $\pm x$ directions along the layer interfaces with different speeds. Although the majority of these cavity modes are well localized within about $\pm 2 D$ from the Ag layer, some components of the waves (possibly those on the branches close to the frequency band of the host SL) spread outside this range.

With the spatial amplitude profiles (or with the magnitude of wavelengths) and group velocities, it is possible to locate the signals corresponding to the points $A$ to $D$ on the SG cavity mode branches. Their center of mass positions are labeled $A^{\prime}$ to $D^{\prime}$ in Figs. 5(c) and also in Fig. 6(b) for $\left|u_{z}\right|$ and $\left|u_{x}\right|$, respectively. We can really see some prominent features at the points $A^{\prime}$ to $D^{\prime}$ indicated. In Figs. 3(a)-3(c) we have shown by open circles and dots the profiles of $u_{z}$ and $-i u_{x}$ obtained by the FDTD simulations along the dashed lines $A^{\prime}$ to $C^{\prime}$ in Figs. 5(c) and 6(b). Except for small structures, the coincidence with the profiles based on the analyti-
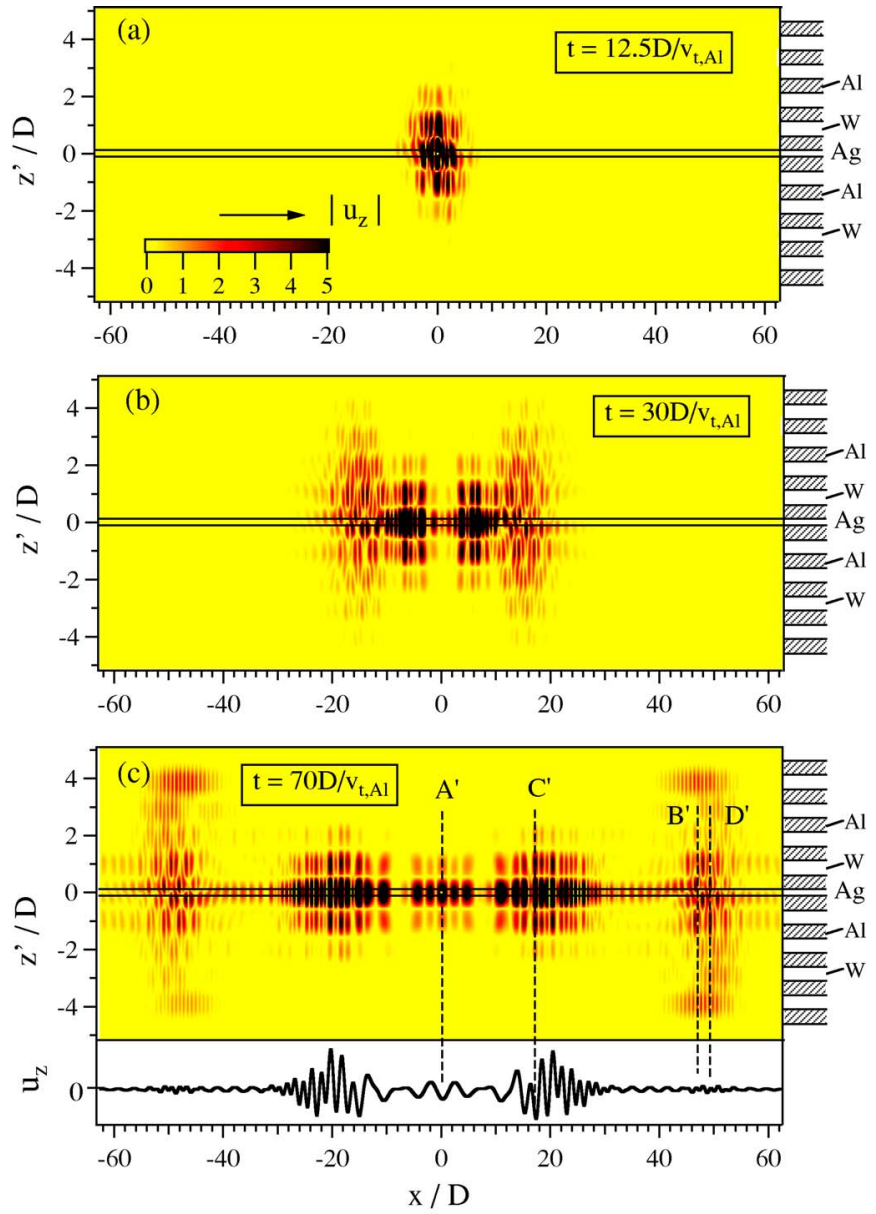

FIG. 5. (Color online) FDTD simulations for the phonon displacement $u_{z}$ patterns in the $x-z$ plane of the $\mathrm{Al} / \mathrm{W}$ multilayers with a Ag cavity layer [the same as for Fig. 2(a)] at $t=(\mathrm{a}) 12.5 \mathrm{D} / v_{t, \mathrm{Al}}$, (b) $30 D / v_{t, \mathrm{Al}}$, and (c) $70 D / v_{t, \mathrm{Al}}$ after the external force $\mathbf{f}$ with $f_{x}=f_{z}$ [Eqs. (63)-(65)] is applied at $x=0$ and $-d_{\mathrm{Ag}} / 3<z^{\prime}<d_{\mathrm{Ag}} / 3$. The darkness corresponds to $\left|u_{z}\right|$. The dashed lines labeled $A^{\prime}$ to $D^{\prime}$ in (c) show the positions of the cavity modes corresponding to the points $A$ to $D$ in Fig. 2(a). They are estimated from the group velocities (the slopes the dispersion curves) at those points on the branches. The bottom part of (c) shows the displacement profile of $u_{z}$ along the center of the cavity $z^{\prime}=0$. The regions in between two parallel horizontal lines are the cavity Ag layers.

cal formulas is excellent at the points $A$ and $C$ including the relative magnitude of $u_{z} /\left(-i u_{x}\right)$. (Here the maximum values of the displacements are scaled to the ones obtained by the analytical formulas.)

However, this is not the case for the smaller component $u_{z}$ at the point $B$ [Fig. 3(b)] and the point $D$ (Fig. 4), that is, the FDTD simulations do not well reproduce the displacement profiles calculated by the analytical formulas. This is possibly due to the fact that the points $B^{\prime}$ and $D^{\prime}$ are close to each other because the group velocities given by the slopes of the branches in Fig. 2(a) at the points $B$ and $D$ have similar magnitudes. So the displacement components at $B^{\prime}$ and $D^{\prime}$ are overlapped to some extent and not completely separated from each other. Also the point $D$ is almost attached to the bulk band (Stoneley band) and hence the associated lattice displacement is extended considerably on either side of the 

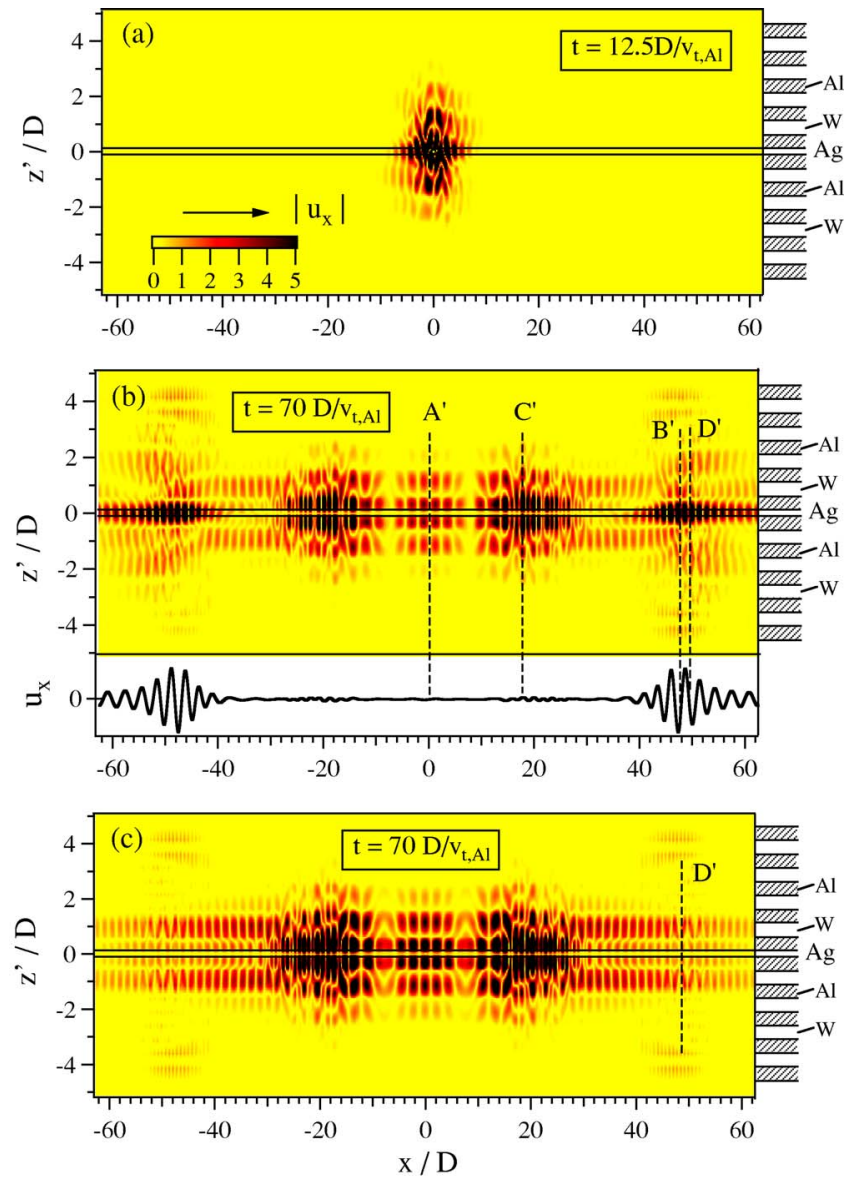

FIG. 6. (Color online) FDTD simulations for the phonon displacement $u_{x}$ patterns in the $x-z$ plane of the $\mathrm{Al} / \mathrm{W}$ multilayers with a Ag cavity layer [the same as for Fig. 2(a)] at $t=(\mathrm{a}) 12.5 \mathrm{D} / v_{t, \mathrm{Al}}$ and (b) $70 D / v_{t, \mathrm{Al}}$. The external force applied for the simulations is the same as for Fig. 5. The darkness corresponds to $\left|u_{x}\right|$. The dashed lines labeled $A^{\prime}$ to $D^{\prime}$ in (b) show the positions of the cavity modes corresponding to the points $A$ to $D$ in Fig. 2(a). The bottom part of (b) shows the displacement profile of $u_{x}$ along the center of the cavity $z^{\prime}=0$. (c) Similar profile of the displacement $u_{x}$ in the $x-z$ plane of the $\mathrm{Al} / \mathrm{W}$ multilayers with a $\mathrm{Ag}$ cavity layer obtained when the external force Eqs. (67)-(69) is given in the $x$ direction to enhance the excitation of the $u_{x}$ component antisymmetric with respect to $z^{\prime}=0$. The regions in between two parallel horizontal lines are the cavity Ag layers.

Ag layer as shown in Figs. 4-6. Thus, the small signal components associated with the point $D$ is not well resolved.

In order to enhance in the simulations the displacement component $u_{x}$ at the point $D$, which is antisymmetric with respect to $z^{\prime}=0\left(z=d_{\mathrm{Ag}} / 2\right)$, we have chosen the external force in the following form:

$$
\begin{gathered}
f_{1}=f_{x}=\left[\Theta\left(z^{\prime}+d_{\mathrm{Ag}} / 2\right)-\Theta\left(z^{\prime}-d_{\mathrm{Ag}} / 2\right)\right] \\
\times \sin \left[\pi\left(\frac{40}{3 D} z^{\prime}+\frac{1}{4}\right)\right] \times g(t), \\
f_{2}=f_{y}=0,
\end{gathered}
$$

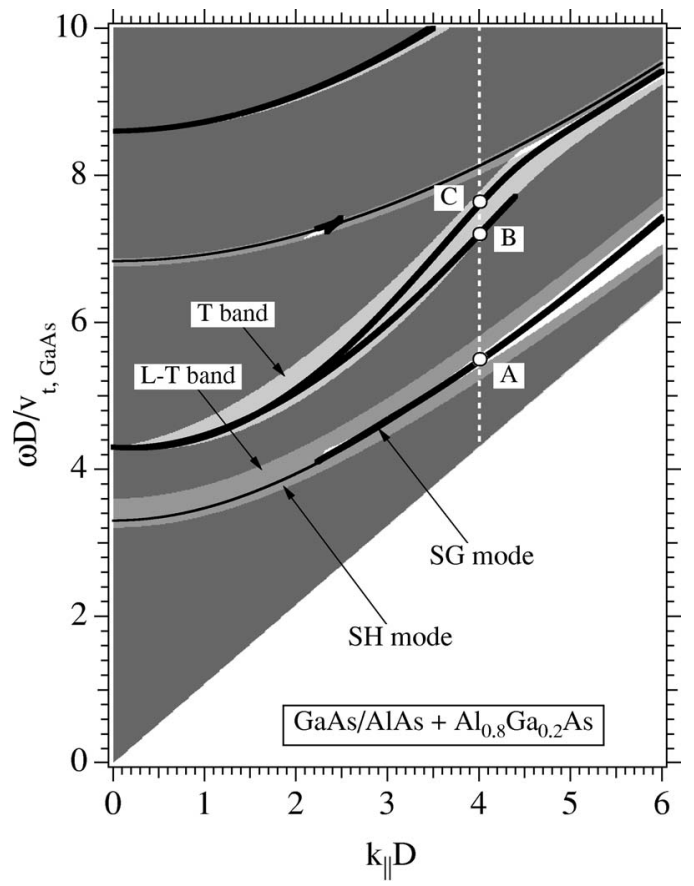

FIG. 7. Band structures and cavity mode branches in the AlAs/GaAs superlattice with $d_{\mathrm{GaAs}}=d_{\mathrm{AlAs}}$. The cavity layer is $\mathrm{Al}_{0.8} \mathrm{Ga}_{0.2} \mathrm{As}$ with thickness $d_{\mathrm{AlGaAs}}=d_{\mathrm{GaAs}} / 2$ sandwiched between AlAs - GaAs and GaAs-AlAs unit cells (the local structure is AlAs - GaAs-AlGaAs-GaAs-AlAs). Each band is discriminated according to the darkness, i.e., the lightest band is the pure $\mathrm{T}$ (SH) band, the second lightest band is the coupled L-T (SG) band, and the darkest band is the overlapped band of both bands. The SG and SH cavity phonon branches are shown by the bold and thin lines, respectively.

$$
f_{3}=f_{z}=0 \text {. }
$$

The simulated result at $t=70 D / v_{t, \mathrm{Al}}$ is shown in Fig. 6(c). The displacement profile $u_{x}$ along the line indicated $D^{\prime}$ is shown in Fig. 4. A good coincidence is seen between the FDTD and analytical results.

\section{B. AlAs/GaAs superlattices with an AlGaAs cavity layer}

The second example of a superlattice we study is a more popular periodic AlAs/GaAs SL which exhibits rather small frequency gaps for vibrational modes due to moderate acoustic impedance matching (Table I) between the constituent semiconductors. Figure 7 depicts the band structures of both the $\mathrm{SG}$ and $\mathrm{SH}$ modes in the AlAs/GaAs SL with $d_{\mathrm{AlAs}}$ $=d_{\mathrm{GaAs}}$ together with the cavity branches. As a cavity layer we have chosen $\mathrm{Al}_{0.8} \mathrm{Ga}_{0.2} \mathrm{As}$ with the thickness $d_{\mathrm{AlGaAs}}$ onehalf of $d_{\mathrm{GaAs}}$. The structure assumed is again symmetric with respect to the cavity later (the layers adjacent to AlGaAs are GaAs). As expected, the band gaps are much smaller than in the case of $\mathrm{Al} / \mathrm{W}$ superlattice and the dispersion curves of the cavity modes do not show any characteristic behaviors for the ranges of the frequency and wave number illustrated.

Figure 8 displays the displacement profiles for three eigenfrequencies at the points marked $A$ to $C$ on the SG cavity branches with the same $k_{\|} D\left(k_{\|} D=4\right)$ as shown in Fig. 

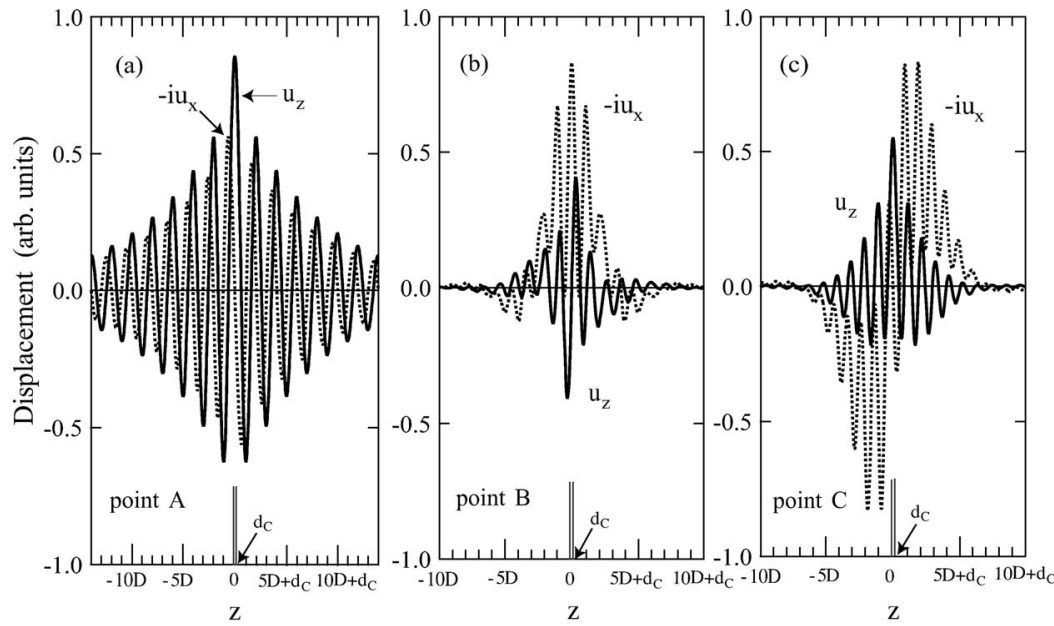

FIG. 8. Displacement profiles of the SG cavity mode in the AlAs/GaAs superlattice with an $\mathrm{Al}_{0.8} \mathrm{Ga}_{0.2}$ As cavity layer with the thickness onehalf of $d_{\mathrm{GaAs}}$ (the same as for Fig. 7). Solid lines are $u_{z}$ (normal to the layer interfaces) and dotted lines are $-i u_{x}$ (parallel to the layer interfaces) calculated with the analytical formula for the points (a) $A$ with $\omega D / v_{t, \text { GaAs }}=5.47$, (b) $B$ with $\omega D / v_{t, \mathrm{GaAs}}=7.19$, and (c) $C$ with $\omega D / v_{t, \mathrm{GaAs}}$ $=7.59$, on the dashed line at $k_{\|} D=4$ in Fig. 7. The positions of the cavity layer are indicated by two parallel vertical lines.

7. Comparing with the case of the cavity modes in the $\mathrm{Al} / \mathrm{W}$ superlattice with an Ag layer, the localization of the wave amplitude near the cavity layer is considerably weak, though it depends on the frequency. We note that the symmetries of $-i u_{x}$ and $u_{z}$ with respect to the cavity layer is interchanged between the points $B$ and $C$ located inside the same frequency gap.

\section{CONCLUSIONS}

We have theoretically studied the phonons confined in a cavity layer embedded in an otherwise perfect, periodic SL. We have given the analytical formulas for determining the eigenfrequencies and displacement vectors of the cavity phonons both for the coupled L-T (SG) and the pure transverse $(\mathrm{SH})$ phonons. According to these formulas, we can find cavity phonon branches inside the frequency gaps of the host superlattice as expected from the general theorem for the vibrations of crystal lattices with a defect. The displacement profiles calculated from our formulas coincide well with those obtained numerically by solving the equations of motion with the FDTD method. Phonons on the cavity branches are confined in the vicinity of the cavity layer but propagate along this layer with the group velocities determined by the slopes of their dispersion curves. This has also been confirmed by the FDTD simulations for the time evolutions of wave packet excited in the assumed system.

It is generally expected that the interactions of these cavity phonons are highly enhanced compared with those of the bulk phonons in the host SLs due to their large amplitudes inside the cavity. Actually, the experiments done by Trigo et al. show that the Raman signal due to cavity phonons is much larger than the ones due to the extended phonons (the so-called "phonon doublet") just above and below the zonecenter gap where the cavity frequency exists. ${ }^{15,16}$ Thus, it should be interesting to study if the electron-phonon interaction and anharmonic phonon-phonon interaction, for instance, of cavity phonons are really enhanced in comparison with the ones for bulk phonons. This is an important subject to be studied separately because these effects in semiconducting and metallic multilayered structures can be observed experimentally with picosecond ultrasonics techniques ${ }^{4}$ and Raman scattering. ${ }^{36}$

\section{ACKNOWLEDGMENTS}

The authors would like to thank Y. Tanaka for useful comments on the manuscript. This work was supported in part by a Grant-in-Aid for Scientific Research from the Ministry of Education, Science and Culture of Japan (Grant No. 17510106).

\section{APPENDIX: SH CAVITY MODE}

In this appendix, we give some notes on the corresponding formulas for the SH cavity phonons (with pure transverse polarization) which are decoupled from the SG cavity phonons in the isotropic approximation. First we note that for this single mode with $\mathbf{k}_{\|} \neq \mathbf{0}$ the wave number $k_{I, Z}$ in each layer is given by $k_{I, z}=\left[\left(\omega / v_{I, t}\right)^{2}-\mathbf{k}_{\|}^{2}\right]^{1 / 2}$ with $I=A, B$, and $C$. The dispersion relation in superlattices becomes $\cos q D$ $=\cos \left(k_{A, z} d_{A}\right) \cos \left(k_{B, z} d_{B}\right)-(1 / 2)\left(C_{44}^{A} k_{A, z} / C_{44}^{B} k_{B, z}\right.$ $\left.+C_{44}^{B} k_{B, z} / C_{44}^{A} k_{A, z}\right) \sin \left(k_{A, z} d_{A}\right) \sin \left(k_{B, z} d_{B}\right)$, where $q$ is the superlattice wave number. With these modifications the formulas given in Ref. 3 for the nonpropagating cavity phonons with $\mathbf{k}_{\|}=\mathbf{0}$ are still applied to the propagating SH cavity mode.

Those formulas for the SH cavity phonons are also obtained straightforwardly from the results of the present work if we regard $\hat{\Gamma}_{I}$ and $\hat{\Phi}_{I}(z)$ as $2 \times 2$ matrices instead of $4 \times 4$ matrices. For example,

$$
\begin{gathered}
\hat{\Gamma}_{A}=\left(\begin{array}{cc}
1 & 1 \\
C_{44}^{A} & -C_{44}^{A}
\end{array}\right), \\
\hat{\Phi}_{A}(z)=\left(\begin{array}{cc}
\exp \left(i k_{A, z} z\right) & 0 \\
0 & \exp \left(-i k_{A, z} z\right)
\end{array}\right),
\end{gathered}
$$

and

$$
\mathbf{A}_{n}=\left(a_{A, n}^{(1)}, a_{A, n}^{(2)}\right)^{t} \equiv\left(a_{A, n}, \tilde{a}_{A, n}\right)^{t} .
$$

Thus the transfer matrices $\hat{T}_{n}^{(1)}-\hat{T}_{n}^{(4)}$ now become scalar quantities. Then the formulations developed in Secs. II and III are still valid for the $\mathrm{SH}$ mode. 
${ }^{1}$ R. E. Camley, B. Djafari-Rouhani, L. Dobrzynski, and A. A. Maradudin, Phys. Rev. B 27, 7318 (1983).

${ }^{2}$ B. Djafari-Rouhani, L. Dobrzynski, O. Hardouin Duparc, R. E. Camley, and A. A. Maradudin, Phys. Rev. B 28, 1711 (1983).

${ }^{3}$ T. Aono and S. Tamura, Phys. Rev. B 58, 4838 (1998).

${ }^{4}$ C. Thomsen, H. T. Grahn, H. J. Maris, and J. Tauc, Phys. Rev. B 34, 4129 (1986).

${ }^{5}$ H. T. Grahn, H. J. Maris, J. Tauc, and B. Abeles, Phys. Rev. B 38, 6066 (1988).

${ }^{6}$ W. Chen, Y. Lu, H. J. Maris, and G. Xiao, Phys. Rev. B 50, 14506 (1994)

${ }^{7}$ B. Perrin, B. Bonello, J.-C. Jeannet, and E. Romatet, Physica B 219\&220, 681 (1996).

${ }^{8}$ H. J. Trodahl, P. V. Santos, G. V. M. Williams, and A. Bittar, Phys. Rev. B 40, 8577 (1989).

${ }^{9}$ E. K. Khourdifi and B. Djafari-Rouhani, Surf. Sci. 211-212, 361 (1989).

${ }^{10}$ D. Bria, E. H. El Boudouti, A. Nougaoui, B. Djafari-Rouhani, and V. R. Velasco, Phys. Rev. B 60, 2505 (1999).

${ }^{11}$ A. I. Murdoch, J. Sound Vib. 50, 1 (1977).

${ }^{12}$ A. A. Maradudin, E. W. Montroll, G. H. Weiss, and I. P. Ipatopva, Theory of Lattice Dynamics in the Harmonic Approximation (Academic, New York, 1971), Chap. 8, and references therein.

${ }^{13}$ J. M. Ziman, Models of Disorder (Cambridge University Press, London, 1979).

${ }^{14}$ S. Tamura, Phys. Rev. B 39, 1261 (1989).

${ }^{15}$ M. Trigo, A. Bruchhausen, A. Fainstein, B. Jusserand, and V. Thierry-Mieg, Phys. Rev. Lett. 89, 227402 (2002).

${ }^{16}$ P. Lacharmoise, A. Fainstein, B. Jusserand, and B. Perrin, Phys. Status Solidi C 1, 2698 (2004).

${ }^{17}$ A. Khelif, B. Djafari-Rouhani, J. O. Vasseur, and P. A. Deymier, Phys. Rev. B 68, 024302 (2003).

${ }^{18}$ Y. Pennec, B. Djafari-Rouhani, J. O. Vasseur, A. Khelif, and P. A. Deymier, Phys. Rev. E 69, 046608 (2004).

${ }^{19}$ S. Fan, P. R. Villeneuve, J. D. Joannopoulos, and H. A. Haus,
Phys. Rev. Lett. 80, 960 (1998).

${ }^{20}$ S. Fan, P. R. Villeneuve, J. D. Joannopoulos, M. J. Khan, C. Manolatou, and H. A. Haus, Phys. Rev. B 59, 15882 (1999).

${ }^{21}$ D. G. Cahill, W. K. Ford, K. E. Goodson, G. D. Mahan, A. Majumdar, H. J. Maris, R. Merlin, and S. R. Phillpot, J. Appl. Phys. 93, 793 (2003).

${ }^{22}$ S. Mizuno and S. Tamura, Phys. Rev. B 45, 13423 (1992).

${ }^{23}$ S. Mizuno and S. Tamura, Phys. Rev. B 53, 4549 (1996).

${ }^{24}$ S. I. Tamura, D. C. Hurley, and J. P. Wolfe, Phys. Rev. B 38, 1427 (1988).

${ }^{25}$ H. Kato and S. Tamura, J. Phys.: Condens. Matter 9, 6791 (1997).

${ }^{26}$ K. Imamura, Y. Tanaka, and S. Tamura, Phys. Rev. B 65, 174301 (2001).

${ }^{27}$ Lord Rayleigh, Proc. London Math. Soc. 17, 4 (1887).

${ }^{28}$ S. Tamura, Phys. Rev. B 56, 12440 (1997).

${ }^{29}$ R. Stoneley, Proc. R. Soc. London, Ser. A 106, 416 (1924).

${ }^{30}$ J. G. Scholte, Mon. Not. R. Astron. Soc. 5, 120 (1947).

${ }^{31}$ G. W. Farnell and E. L. Adler, Physical Acoustic (Academic, New York, 1972), Vol. IX.

${ }^{32}$ B. A. Auld, Acoustic Fields and Waves in Solids (Wiley, New York, 1973).

${ }^{33}$ A. Taflove, in Advances in Computational Electrodynamics: The Finite-Difference Time-Domain Method, edited by C. M. Soukoulis (Artech House, London, 1998).

${ }^{34}$ Y. Tanaka, Y. Tomoyasu, and S. Tamura, Phys. Rev. B 62, 7387 (2000).

${ }^{35}$ Y. Tanaka, M. Takigahira, and S. Tamura, Phys. Rev. B 66, 075409 (2002)

${ }^{36}$ C. Colvard, R. Merlin, M. V. Klein, and A. C. Gossard, Phys. Rev. Lett. 45, 298 (1980); C. Colvard, T. A. Gant, M. V. Klein, R. Merlin, R. Fischer, H. Morkoc, and A. C. Gossard, Phys. Rev. B 31, 2080 (1985); B. Jusserand, D. Paquet, and A. Regreny, ibid. 30, 6245 (1984). 